\title{
Higher Dimensional Charged Black Hole Solutions in $f(R)$ Gravitational Theories
}

\author{
G. G. L. Nashed $\mathbb{I D}^{1,2,3}$ \\ ${ }^{1}$ Centre for Theoretical Physics, The British University in Egypt, P.O. Box 43, Cairo 11837, Egypt \\ ${ }^{2}$ Mathematics Department, Faculty of Science, Ain Shams University, Cairo, Egypt \\ ${ }^{3}$ Egyptian Relativity Group (ERG), Cairo University, Giza, Egypt \\ Correspondence should be addressed to G. G. L. Nashed; nashed@bue.edu.eg
}

Received 28 July 2017; Revised 31 October 2017; Accepted 9 November 2017; Published 21 January 2018

Academic Editor: Elias C. Vagenas

Copyright (c) 2018 G. G. L. Nashed. This is an open access article distributed under the Creative Commons Attribution License, which permits unrestricted use, distribution, and reproduction in any medium, provided the original work is properly cited. The publication of this article was funded by SCOAP ${ }^{3}$.

\begin{abstract}
We present, without any assumption, a class of electric and magnetic flat horizon $D$-dimension solutions for a specific class of $f(R)=R+\alpha R^{2}$, all of which behave asymptotically as Anti-de-Sitter spacetime. The most interesting property of these solutions is that the higher dimensions black holes, $D>4$, always have constant electric and magnetic charges in contrast to what is known in the literature. For $D=4$, we show that the magnetic field participates in the metric on equal foot as the electric field participates. Another interesting result is the fact that the Cauchy horizon is not identical with the event horizon. We use Komar formula to calculate the conserved quantities. We study the singularities and calculate the Hawking temperature and entropy and show that the first law of thermodynamics is always satisfied.
\end{abstract}

\section{Introduction}

The most effective gravitational theory in the last century is the theory of general relativity (GR). This theory is a fully accepted one that depicts the macroscopic geometrical properties of spacetime. Using an isotropic and homogeneous symmetry, the field equations of GR give Friedmann equations which depict the evolution of the universe with radiation and then matter dominated epochs. However, recent observations indicate that our universe goes through a phase of accelerated expansion [1-3]. This fact cannot be explained in the frame of GR using ordinary matter as a source. Another issue that GR cannot explain is the cosmological era which is known as inflation [4]. This phase of the universe is believed to have occurred before the radiation era which could relax some issues of cosmology like horizon, flatness singularities, and so on [5]. Moreover, using baryonic matter, GR is not able to discuss the observed density limited by the fitting of the standard $\Lambda$ cold dark matter $(\Lambda \mathrm{CDM})$ of the Wilkinson Microwave Anisotropy Probe results for 7 years of observations data (WMAP7) [6], the recent measurements of Baryon Acoustic Oscillations (BAO) [7], and the Hubble constant $H_{0}$ [8]. Therefore, GR needs to impose an extra component known as the dark matter (DM) which constitutes about $23 \%$ of the energy content of the universe [7]. In spite of the fact that there are many possible roots of such component [9-18], DM is assumed in a form of thermal relics which naturally freeze-out with the right abundance in the extensions of the standard model of particles [19-31]. Coming experiments enable us to distinguish between large number of candidates and model by direct and indirect detection prepared for their search [32-34], or even at large hadron collider (LHC) where they could be produced [35-42].

Another puzzling issue is the one of the accelerated expansion of our universe. Many explanations have been setup to demonstrate such phenomena. Among these explanations is the one which assumes the validity of GR and suggests the presence of extra fluid called dark energy (DE). The equation of state of DE takes the form $p=\omega \rho$ (where $p$, $\rho$, and $\omega$ are pressure, density, and a dimensionless parameter, 
resp.) with $\omega<-1 / 3$ to create an accelerated cosmic expansion era [43-45]. There is another model which could explain the DE which includes the cosmological constant in the field equations of GR and assumes the equation of state to have the form $\omega=-1$. However, such model suffers from the discrepancy which comes from the fact that if we postulate the cosmological constant to represent the quantum vacuum energy then its value has higher orders of magnitude than those of observations [46]. It has been shown that, in the Palatini formalism of $f(R)=R-\alpha^{2} / 3 R+R^{2} / 3 \beta, \alpha$ and $\beta$ are dimension parameters, the $R^{2}$ term cannot lead to an early time inflation [47].

To overcome the problem of acceleration a modification of GR has been considered by modifying Einstein-Hilbert action [48-62]. Some examples of these modifications are as follows:

(i) Brans-Dicke theory whose interaction is considered by GR tensor and scalar field [63]

(ii) String theory which include Gauss-Bonnet term [64]

(iii) Lovelock theories which are of second derivatives at most [65].

(iv) The $f(T)$ gravitational theory whose field equations are of second order [66-80]

(v) The one known as $f(R)$ theories which we focused the present study on [81]

It is shown that $f(R)$ gravitational theories are able to describe the whole story of cosmology starting from inflation to the present accelerated expansion epoch [82]. Many applications of $f(R)$ gravitational theories have been carried out $[83,84]$. Also local tests on $f(R)$ have been achieved to constrain $f(R)$ theories [85-88]. To study modified theories of gravity one requires to assure or reject their validity by deriving solutions that could investigate the evolution of the universe $[89,90]$ and the occurrence of GR astrophysical prediction. There are many BH derived in the frame of $f(R)$ by assuming a constant scalar curvature, $R=R_{0}$ [91-93]. It is the aim of the present study to abandon this condition $R=R_{0}$ in a class of $f(R)=R+\alpha R^{2}$ and try to derive $D$-dimension solutions for a flat horizon metric spacetime.

The arrangements of this study are as follows: In Section 2, gradients elements of Maxwell- $f(R)$ gravitational theory are presented. In Section 3, a metric spacetime with one unknown and two unknown functions is presented and applied to the charged field equation of $f(R)$. Exact classes of charged black holes are derived in Section 3. In Section 4, the relevant physics of these classes is discussed by calculating the singularities. In Section 5, we calculate the conserved charges related to each class by using Komar method. In Section 6, we calculate the thermodynamical quantities like Hawking temperature and entropy, and so on. Also in this section, we have shown that the first law of thermodynamics is always satisfied for all the solutions derived in this study. The main results are discussed in the final section.

\section{Fundamentals of Maxwell- $f(R)$ Gravitational Theories}

The Lagrangian of $f(R)$ theory has the form

$$
\mathscr{L}:=\mathscr{L}_{g}+\mathscr{L}_{\text {em }},
$$

with $\mathscr{L}_{g}$ representing the gravitational Lagrangian given by

$$
\mathscr{L}_{g}:=\frac{1}{2 \kappa} \int d^{D} x \sqrt{-g}(f(R)-\Lambda),
$$

with $\Lambda$ being the cosmological constant and $\kappa$ being the $D$ dimensional gravitational constant defined as $\kappa=2(D-$ 3) $\Omega_{D-1} G_{D}$, where $G_{D}$ is the Newton's constant in $D$ dimensions (the units for the $D$-dimensional gravitational constant are $G_{D}=G_{4} L_{D-4}$, where $G_{4}$ is the gravitational constant in 4-spacetime dimensions and $L$ is a unit of length). Here $\Omega_{D-1}$ refers to the volume of $(D-1)$-dimensional unit sphere that is defined as

$$
\Omega_{D-1}=\frac{2 \pi^{(D-1) / 2}}{\Gamma((D-1) / 2)},
$$

where $\Gamma$ is the gamma function of the argument (for $D=4$, one gets $\left.2(D-3) \Omega_{D-1}=8 \pi\right), R$ is the Ricci scalar of the spacetime, $g$ is the determinant of the metric, and $f(R)$ is the analytic function of the considered theory. In this study $L_{e m}$ refers to the action of Maxwell field that is defined as

$$
L_{e m}:=-\frac{1}{2} F \wedge^{\star} F, \quad \text { where } F=d A,
$$

with $A=A_{\mu} d x^{\mu}$ being the 1-form electromagnetic potential [94].

By carrying out variations of (2) with respect to the metric tensor $g_{\mu \nu}$ and the vector potential $A_{\mu}$ one can obtain the following field equations of $f(R)$ gravitational theory $[95,96]$ :

$$
\begin{aligned}
& R_{\mu \nu} f_{R}-\frac{1}{2} g_{\mu \nu} f(R)-2 g_{\mu \nu} \Lambda+g_{\mu \nu} \square f_{R}-\nabla_{\mu} \nabla_{\nu} f_{R} \\
& \quad=2 \kappa T_{\mu \nu}, \\
& \partial_{\nu}\left(\sqrt{-g} F^{\mu \nu}\right)=0,
\end{aligned}
$$

with $R_{\mu \nu}$ being the Ricci tensor defined by

$$
\begin{aligned}
R_{\mu \nu} & =R_{\mu \rho \nu}^{\rho}=\partial_{\rho} \Gamma_{\mu \nu}^{\rho}-\partial_{\nu} \Gamma_{\mu \rho}^{\rho}+\Gamma_{\rho \beta}^{\rho} \Gamma_{\mu \nu}^{\beta}-\Gamma_{\nu \beta}^{\rho} \Gamma_{\mu \rho}^{\beta} \\
& =2 \Gamma_{\mu[\nu, \rho]}^{\rho}+2 \Gamma_{\beta[\rho}^{\rho} \Gamma_{\nu] \mu}^{\beta},
\end{aligned}
$$

where $\Gamma_{\mu \nu}^{\rho}$ is the Christoffel symbols second kind and the square brackets mean

$$
A_{[\mu, v]}=\frac{1}{2}\left(A_{\mu, \nu}-A_{\nu, \mu}\right) .
$$

The D'Alembert operator $\square$ is defined as $\square=\nabla_{\alpha} \nabla^{\alpha}$, where $\nabla_{\alpha} V^{\beta}$ is the covariant derivatives of the vector $V^{\beta}$ and $f_{R}=$ $d f(R) / d R$. In this study $T_{\mu \nu}$ is defined as

$$
T_{\mu \nu}:=g_{\rho \sigma} F^{\nu \rho} F_{\mu}{ }^{\sigma}-\frac{1}{4} \delta_{\mu}{ }^{\nu} g^{\lambda \rho} g^{\epsilon \sigma} F_{\lambda \epsilon} F_{\rho \sigma},
$$


which is the energy momentum tensor of the electromagnetic field.

The trace of (5) yields

$$
R f_{R}-2 f(R)-8 \Lambda+3 \square f_{R}=0 .
$$

Now we are going to discuss some basic property of the above $f(R)$ theories.

\section{1. $f(R)$-Gravitational Theories and Their Viable Conditions.} The most important conditions and restrictions [81] that are usually put on $f(R)$ gravitational theories to give consistency on both of gravitational and cosmological evolutions are as follows.

(a) The first condition is given by

$$
f^{\prime \prime}(R) \geq 0, \quad \text { when } R \geq f^{\prime \prime}(R)
$$

which represents the stability condition for curvature [97]. Condition (11) represents the existence of a matter dominated era in cosmological evolution. The relevant physics of (11) is that if the constant,

$$
G_{\text {eff }}=\frac{G}{f^{\prime}(R)},
$$

has a defined value, then $d G_{\text {eff }} / d R$ is fixed by the sign of $f^{\prime \prime}(R)$.

(b) The condition $f^{\prime}(R)>0$ guarantees the positivity of the effective gravitational constant. This condition from quantum point of view avoids the graviton from becoming a ghost $[98,99]$.

(c) The condition $f^{\prime}(R)<0$ ensures the recovery of GR behavior at early times.

\section{Analytic Solution in $f(R)=R+\alpha R^{2}$}

In this section, we are going to apply the field equations of Maxwell- $f(R)$ with cosmological constant to two different metric spacetimes having flat horizon:

3.1. Flat Horizon Metric with One Variable. Let us assume the first metric spacetime possessing one unknown function has the form

$$
d s^{2}=-a(r) d t^{2}+\frac{1}{a(r)} d r^{2}+r^{2} \sum_{i=1}^{D-2} d \theta^{2},
$$

where $a(r)$ is an unknown function of the radial coordinate, $r$. Using (13) we get the Ricci scalar in the form

$$
R=-\frac{r^{2} a^{\prime \prime}+2(D-2) r a^{\prime}+(D-2)(D-3) a}{r^{2}}
$$

where $a^{\prime}=d a(r) / d r$ and $a^{\prime \prime}=d^{2} a(r) / d r^{2}$. The nonvanishing components of the Maxwell- $f(R)$ field equations, (5) and (6), when $f(R)=R+\alpha R^{2}$ take the form (the detailed calculations of the Ricci curvature tensor are given in Appendix B)

$$
\begin{aligned}
\aleph_{t t} & =\frac{1}{2 r^{4}}\left(\left[\alpha r^{4} a^{\prime \prime 2}-4 r^{4} \Lambda+2 r^{4} q^{\prime 2}+2 s_{\theta_{D-4}}^{2}\right]-(D\right. \\
& -2) r^{2}\left[2 r \alpha a^{\prime} a^{\prime \prime}-r a^{\prime}-a(D-3)\right]-4 \alpha r^{2} a a^{\prime \prime}(D \\
& -2)(3 D-11)-4 \alpha r a a^{\prime}\left[D^{3}-10 D^{2}+24 D-4\right] \\
& -4 \alpha a^{2}(4 D-11)-2 \alpha r^{3}\left\{a^{\prime \prime \prime}\left[r a^{\prime}+6 a(D-2)\right]\right. \\
& \left.\left.+2 r a a^{\prime \prime \prime \prime}\right\}-2 \alpha r^{2} a^{\prime 2}(D-2)(D-5)\right)=0, \\
\aleph_{r r} & =\frac{1}{2 r^{4}}\left(\left[\alpha r^{4} a^{\prime \prime 2}-4 r^{4} \Lambda+2 r^{4} q^{\prime 2}+2 s_{\theta_{D-4}}^{2}\right]-(D\right. \\
& -2) r^{2}\left[2 r \alpha a^{\prime} a^{\prime \prime}-r a^{\prime}-a(D-3)\right]-8 \alpha r^{2} a a^{\prime \prime}(D \\
& -2)^{2}-4 \alpha r a a^{\prime}\left[D^{3}-7 D^{2}+9 D+8\right]-4 \alpha a^{2}(2 D \\
& -1)(2 D-7)-2 \alpha r^{3} a^{\prime \prime \prime}\left[r a^{\prime}+2 a(D-2)\right] \\
& \left.-2 \alpha r^{2} a^{\prime 2}(D-2)(D-5)\right)=0, \\
& -4 \alpha r^{3}\left\{a^{\prime \prime \prime}\left[r a^{\prime}+a(3 D-7)\right]+r a a^{\prime \prime \prime \prime}\right\} \\
& +4 r^{4} \Lambda+2 r^{4} q^{\prime 2}+2 s_{\theta_{D-4}}^{2}+4 \alpha r^{3} a^{\prime} a^{\prime \prime}(3 D-7) \\
\aleph_{\theta_{1} \theta_{1}} & =S_{\theta_{2} \theta_{2}}=\cdots\left[8\left\{D^{2}-13 D+34\right\} r a^{\prime}\right. \\
& -2(D-3) r^{3} a^{\prime}-r^{2} \alpha a^{\prime 2}(D-2)(2 D-9) \\
& \\
&
\end{aligned}
$$

where $s_{\theta_{D-4}}=d s\left(\theta_{D-4}\right) / d \theta_{D-4}$ and $q^{\prime}=d q(r) / d r$ with $q(r)$ and $s(\theta)$ being two unknown functions related to the electric and magnetic charges of the system and defined from the gauge potential as

$$
A \stackrel{\text { def. }}{=} q(r) d t+s\left(\theta_{D-4}\right) d \theta_{D-4} \text {. }
$$

The solution of the differential equations (15) has the form

$$
\begin{aligned}
& q(r)=c_{1}+\delta_{4}{ }^{4} \frac{c_{2}}{r}, \\
& s\left(\theta_{D-4}\right)=c_{3}+\delta_{4}{ }^{4} c_{4} \theta_{D-4}, \\
& a(r)=\left(\frac{2 r^{2} \Lambda}{3}+\frac{c_{5}}{r}+\frac{c_{2}{ }^{2}+c_{4}{ }^{2}}{r^{2}(1-16 \alpha \Lambda)}\right) \delta_{4}{ }^{4} \\
& +\delta_{h}{ }^{h}\left[\frac{(D-2) r^{D-1}\left[1 \pm \sqrt{1-16 D(D-4) \alpha \Lambda /(D-2)^{2}}\right]}{2 D(D-1)(D-4) \alpha r^{D-3}}\right. \\
& \left.+\frac{c_{6}}{r^{D-3}}\right],
\end{aligned}
$$


where $h \geq 5$ and $c_{i}, i=1 \cdots 6$ are constants. Solution (17) is decomposed into two parts one for $D=4$ and the other for $D \geq 0$. The reason for this decomposition is the electromagnetic field. In the four dimensions there is a charged solution; however, for $D \geq 5$ there is no charged solution. In fact this is in conflict with the spherically symmetric case [91]. It is important to mention here that solution (17) when $\alpha=0$ will reduce to the well known AdS/dS solution in the case of $D=4$. However, in the case of $D>4$ this solution does not allow the parameter $\alpha$ to be vanishing; therefore, it has no analogue in GR. In the noncharged case and when $D=4$ solution (17) coincides with what is know in GR. However, when $D>4$ and as long as $\alpha$ and $\Lambda$ have no relation between them solution (17) has no analogue in GR but when $\Lambda=1 / 16 \alpha$ it will coincide with what is known in GR; that is, it behaves as Ads/dS.

The metric of solutions (17) has the form

$$
\begin{aligned}
d s^{2} & =-\left\{\left(\frac{2 r^{2} \Lambda}{3}+\frac{c_{5}}{r}+\frac{c_{2}{ }^{2}+c_{4}{ }^{2}}{r^{2}(1-16 \alpha \Lambda)}\right) \delta_{4}{ }^{4}+\delta_{h}{ }^{h}\left[\frac{(D-2) r^{D-1}\left[1 \pm \sqrt{1-16 D(D-4) \alpha \Lambda /(D-2)^{2}}\right]}{2 D(D-1)(D-4) \alpha r^{D-3}}+\frac{c_{6}}{r^{D-3}}\right]\right\} d t^{2} \\
& +\frac{d r^{2}}{\left(2 r^{2} \Lambda / 3+c_{5} / r+\left(c_{2}{ }^{2}+c_{4}{ }^{2}\right) / r^{2}(1-16 \alpha \Lambda)\right) \delta_{4}{ }^{4}+\delta_{h}{ }^{h}\left[(D-2) r^{D-1}\left[1 \pm \sqrt{1-16 D(D-4) \alpha \Lambda /(D-2)^{2}}\right] / 2 D(D-1)(D-4) \alpha r^{D-3}+c_{6} / r^{D-3}\right]} \\
+ & r^{2} \sum_{i=1}^{D-2} d \theta^{2}{ }_{i} .
\end{aligned}
$$

3.2. Metric with Two Variables. The metric of a flat horizon with two unknown functions has the form

$$
d s^{2}=-a(r) d t^{2}+\frac{1}{b(r)} d r^{2}+r^{2} \sum_{i=1}^{D-2} d \theta_{i}^{2},
$$

where $a(r)$ and $b(r)$ are two unknown functions of the radial coordinate, $r$. Using (19) we get the Ricci scalar in the form

$$
\begin{aligned}
R & =-\left(\frac{a^{\prime} b^{\prime}}{2 a}+\frac{b a^{\prime \prime}}{a}-\frac{b a^{\prime 2}}{2 a^{2}}+\frac{(D-2) b a^{\prime}}{a r}\right. \\
& \left.+\frac{(D-2) b^{\prime}}{r}+\frac{(D-2)(D-3) b}{r^{2}}\right) .
\end{aligned}
$$

Using (19) we get the nonvanishing components of the field equations, (5) and (6), when $f(R)=R+\alpha R^{2}$ in the form

$$
\begin{aligned}
& \aleph_{t t}=\frac{1}{8 r^{4} a^{4}}\left(36 \alpha r^{4} a^{2} b^{2} a^{\prime \prime 2}-2 \alpha r^{3} b\left(a a^{\prime 3}\left[29 r b^{\prime}+26(D-2) b\right]+8 r b a^{3} a^{\prime \prime \prime \prime}\right)+8 a^{3}\left[a s_{\theta_{D-4}}^{2}+r^{4} b q^{\prime 2}\right]+49 \alpha r^{4} b^{2} a^{\prime 4}\right. \\
& +\alpha r^{2} a^{\prime 2} a^{2}\left\{2 b(D-2)\left[2(5 D-18) b+30 r b^{\prime}\right]+9 r^{2} b^{\prime 2}\right\}-8 \alpha a^{2} b r^{3}\left\{a b^{\prime \prime \prime}\left[2(D-2) a+r a^{\prime}\right]\right. \\
& \left.-2 a^{\prime \prime \prime}\left[3 r b a^{\prime}-a\left(2(D-2) b+3 r b^{\prime}\right)\right]\right\}+4 r^{2} \alpha a a^{\prime \prime}\left\{r b a^{\prime}\left[a\left\{27 r b^{\prime}+22(D-2) b\right\}-29 r b a^{\prime}\right]\right. \\
& \left.-a^{2}\left[16(D-2) r b b^{\prime}+3 b^{\prime 2}+4(D-2)(D-4) b^{2}\right]\right\}-4 r^{2} a^{2} \alpha b^{\prime \prime}\left[2(D-2) a^{2}\left(2(2 D-7) b+r b^{\prime}\right)\right. \\
& \left.+r a a^{\prime}\left[6(D-2) b+r b^{\prime}\right]-6 r^{2} b a^{\prime 2}\right]-8(D-2) r \alpha a^{3} a^{\prime}\left[r b^{\prime}+2(D-4) b\right]\left[r b^{\prime}-b\right]-4 a^{3}(D-2) \\
& \cdot r a b^{\prime}\left[(3 D-10) \alpha r b^{\prime}-r^{2}-\{2(3 D-11)(D-4)+4(3 D-10)(D-5)\} \alpha b\right. \\
& \left.\left.+a\left(4 r^{4} \Lambda-(D-2)(D-3) r^{2} b-4(4 D-11) \alpha b^{2}\right)\right]\right)=0 \\
& \aleph_{r r}=\frac{1}{8 r^{4} a^{4}}\left(4 \alpha r^{4} a^{2} b^{2} a^{\prime \prime 2}+2 \alpha r^{3} b a a^{\prime 3}\left[3 r b^{\prime}-4(D-2) b\right]+8 a^{3}\left[a s_{\theta_{D-4}}^{2}+r^{4} b q^{\prime 2}\right]-7 \alpha r^{4} b^{2} a^{\prime 4}+\alpha r^{2} a^{\prime 2} a^{2}\left\{r^{2} b^{\prime 2}\right.\right. \\
& \left.+4 b(D-2)\left[(3 D-4) b+r b^{\prime}\right]\right\}-8 \alpha a^{2} b^{2} r^{3} a^{\prime \prime \prime}\left[2 a(D-2)+r a^{\prime}\right] \\
& -4 r^{2} \alpha a b\left\{a^{\prime \prime}\left(r a^{\prime}\left[2 a\left\{r b^{\prime}-3(D-2) b\right\}-3 r b a^{\prime}\right]+4(D-2) a^{2}\left[r b^{\prime}+(D-2) b\right]\right)+a b^{\prime \prime}\left[2(D-2) a+r a^{\prime}\right]^{2}\right\} \\
& -4(D-2) r a^{3} a^{\prime}\left[2(3 D-8) \alpha r b b^{\prime}-r^{2} b-4 \alpha b^{2}-\alpha r^{2} b^{\prime 2}\right]+4 a^{3}\left\{(D-2)^{2} \alpha r^{2} a b^{\prime 2}\right. \\
& \left.\left.-a\left[4 r^{4} \Lambda+(D-2)\left[4 r b \alpha(D-2)(D-4)-(D-3) r^{2} b-4(4 D-9) \alpha b^{2}\right]\right]\right\}\right)=0 \text {, }
\end{aligned}
$$




$$
\begin{aligned}
& \aleph_{\theta_{1} \theta_{1}}=\aleph_{\theta_{2} \theta_{2}}=\cdots=\aleph_{\theta_{D-2} \theta_{D-2}}=\frac{1}{8 r^{4} a^{4}}\left(28 \alpha r^{4} a^{2} b^{2} a^{\prime \prime 2}-2 \alpha r^{3} b\left(a a^{\prime 3}\left[23 r b^{\prime}+6(3 D-7) b\right]+8 r b a^{3} a^{\prime \prime \prime \prime}\right)\right. \\
& -8 a^{3}\left[a s_{\theta_{D-4}}^{2}+r^{4} b q^{\prime 2}\right]+39 \alpha r^{4} b^{2} a^{\prime 4} \\
& +\alpha r^{2} a^{\prime 2} a^{2}\left\{2 b(D-2)\left[\{2(D-2)(D-5)+(2 D-5)(D-4)\} b+4(3 D-7) r b^{\prime}\right]+7 r^{2} b^{\prime 2}-\frac{2 r^{2} b}{\alpha}\right\} \\
& -8 \alpha a^{2} b r^{3}\left\{a b^{\prime \prime \prime}\left[\left(2(D-2) a+r a^{\prime}\right)\right]-a^{\prime \prime \prime}\left[5 r b a^{\prime}-a\left(2(2 D-5) b+6 r b^{\prime}\right)\right]\right\} \\
& +4 r^{2} \alpha a a^{\prime \prime}\left\{r b a^{\prime}\left[a\left\{2(8 D-19) b+22 r b^{\prime}\right\}-23 r b a^{\prime}\right]\right. \\
& \left.-a^{2}\left[2(9 D-22) r b b^{\prime}+3 r^{2} b^{\prime 2}-4[2(D-3)(D-5)-(D-4)] b^{2}-\frac{r^{2} b}{\alpha}-8 r^{2} a^{2} b b^{\prime \prime}\right]\right\} \\
& -4 r^{2} a^{2} \alpha b^{\prime \prime}\left[2(D-2) a^{2}\left(4(D-4) b+r b^{\prime}\right)+r a a^{\prime}\left[2(4 D-9) b+r b^{\prime}\right]-5 r^{2} b a^{\prime 2}\right]+2 r a^{3} a^{\prime}\left[r^{3} b^{\prime}+2(D-3) r^{2} b\right. \\
& \left.-4[2(D-1)(D-5)+(D-4)(3 D-11)] \alpha r b b^{\prime}-2(3 D-7) r^{2} \alpha b^{\prime 2}+8(5 D-19) \alpha b^{2}\right] \\
& -4 a^{3}\left[3(D-4)(D-2) \alpha r^{2} a b^{2}-(D-3) r a b^{\prime}\left[r^{2}+4(2 D-1) \alpha b\right]\right. \\
& \left.\left.+a\left(4 r^{4} \Lambda-\left\{(D-4)(D-3) r^{2} b-2[(D-6)(D-3)(2 D-1)-D(D-4)(D-5)] \alpha b^{2}\right\}\right)\right]\right)=0
\end{aligned}
$$

The above system of differential equations (21) has the following solution:

$$
\begin{aligned}
& \text { (1) } q(r)=c_{7}, \\
& s\left(\theta_{D-4}\right)=c_{8}+\delta_{4}{ }^{4} c_{9} \theta_{D-4}, \\
& a(r)=\frac{c_{10}\left(r\left[2 r^{3} \Lambda+3 c_{11}\right](16 \alpha \Lambda-1)-3 c_{12}{ }^{2}\right)}{r^{2}} \delta_{4}{ }^{4}+\delta_{h}{ }^{h} c_{20} \\
& \cdot \frac{(D-2) r^{D-1}\left[\sqrt{1-16 D(D-4) \alpha \Lambda /(D-2)^{2}} \pm 1\right] \pm c_{13}}{r^{D-3}}, \\
& b(r)=\frac{r\left[2 r^{3} \Lambda+3 c_{11}\right](16 \alpha \Lambda-1)-3 c_{12}{ }^{2}}{3 r^{2}(16 \alpha \Lambda-1)} \delta_{4}{ }^{4} \\
& +\delta_{h}{ }^{2}\left[\frac{(D-2) r^{D-1}\left[\sqrt{1-16 D(D-4) \alpha \Lambda /(D-2)^{2}} \pm 1\right]}{2 D(D-1)(D-4) \alpha r^{D-3}}\right. \\
& \left. \pm \frac{c_{13}}{r^{D-3}}\right],
\end{aligned}
$$

(2) $q(r)=c_{14}+\delta_{4}{ }^{4} \frac{c_{15}}{r}$,

$s\left(\theta_{D-4}\right)=c_{16}+\delta_{4}{ }^{4} c_{17} \theta_{D-4}$,

$$
\begin{aligned}
a(r) & =\frac{{c_{15}}^{2}\left(2 \Lambda r^{4}+3\left(c_{18}+r c_{19}\right)\right)}{3 r^{2}\left(c_{17}{ }^{2}+c_{18}[16 \alpha \Lambda-1]\right)} \delta_{4}{ }^{4}+\delta_{h}{ }^{h} c_{20} \\
\cdot & \frac{(D-2) r^{D-1}\left[\sqrt{1-16 D(D-4) \alpha \Lambda /(D-2)^{2}} \pm 1\right] \pm c_{13}}{r^{D-3}}, \\
b(r) & =\frac{3\left(c_{18}+r c_{19}\right)+2 r^{2} \Lambda}{3 r^{2}} \delta_{4}{ }^{4} \\
+ & \delta_{h}{ }^{h}\left[\frac{(D-2) r^{D-1}\left[\sqrt{1-16 D(D-4) \alpha \Lambda /(D-2)^{2}} \pm 1\right]}{2 D(D-1)(D-4) \alpha r^{D-3}}\right. \\
\pm & \left.\frac{c_{13}}{r^{D-3}}\right]
\end{aligned}
$$

where $h \geq 5$. We must mention here that solution (22) has the same property of solution (17); that is, when $\alpha=0$ it will reduce to the well known AdS/dS solution in the case of $D=$ 4. However, in the case of $D>4$ this solution does not allow the parameter $\alpha$ to be vanishing; therefore, this solution has no analogue in GR. In the noncharged case and when $D=4$ and $\alpha \neq 0$ solution (22) coincides with what is known in GR. However, when $D>4$ and as long as $\alpha$ and $\Lambda$ have no relation between them solution (22) has no analogue in GR but when $\Lambda=1 / 16 \alpha$ it will coincide with GR solution that behaves as Ads/dS. The metric spacetimes of solutions (22) have the form 
(1) $d s^{2}=-\left[\frac{c_{10}^{2}\left(32 r^{4} \alpha \Lambda^{2}+48 c_{11} \alpha \Lambda r-2 r^{4} \Lambda+3 c_{12}^{2}+3 c_{11} r\right)}{r^{2}} \delta_{4}^{4}\right.$

$\left.+\delta_{h}{ }^{h} c_{20} \frac{(D-2) r^{D-1}\left[\sqrt{1-16 D(D-4) \alpha \Lambda /(D-2)^{2}} \pm 1\right] \pm c_{13}}{r^{D-3}}\right] d t^{2}$

$+\left\{\frac{32 r^{4} \alpha \Lambda^{2}+48 c_{11} \alpha \Lambda r-2 r^{4} \Lambda-3 c_{12}^{2}-3 c_{11} r}{3 r^{2}(16 \alpha \Lambda-1)} \delta_{4}^{4}\right.$

$\left.+\delta_{h}{ }^{h}\left[\frac{(D-2) r^{D-1}\left[1 \pm \sqrt{1-16 D(D-4) \alpha \Lambda /(D-2)^{2}}\right]}{2 D(D-1)(D-4) \alpha r^{D-3}} \pm \frac{c_{13}}{r^{D-3}}\right]\right\}^{-1} d r^{2}+r^{2} \sum_{i=1}^{D-2} d \theta^{2}$,

(2) $d s^{2}=-\left(\frac{c_{15}{ }^{2}\left(2 \Lambda r^{4}+3\left(c_{18}+r c_{19}\right)\right)}{3 r^{2}\left(c_{17}{ }^{2}+c_{18}[16 \alpha \Lambda-1]\right)} \delta_{4}{ }^{4}+\delta_{h}{ }^{h} c_{20} \frac{(D-2) r^{D-1}\left[1 \pm \sqrt{1-16 D(D-4) \alpha \Lambda /(D-2)^{2}}\right] \pm c_{13}}{r^{D-3}}\right) d t^{2}$

$+\left[\frac{3\left(c_{18}+r c_{19}\right)+2 r^{2} \Lambda}{3 r^{2}} \delta_{4}{ }^{4}+\delta_{h}{ }^{h}\left\{\frac{(D-2) r^{D-1}\left[\sqrt{1-16 D(D-4) \alpha \Lambda /(D-2)^{2}} \pm 1\right]}{2 D(D-1)(D-4) \alpha r^{D-3}} \pm \frac{c_{13}}{r^{D-3}}\right\}\right]^{-1} d r^{2}$

$+r^{2} \sum_{i=1}^{D-2} d \theta^{2}$

where $c_{i}, i=6 \cdots 19$ are constants.

\section{Physical Properties of the Analytic Solutions}

$+\frac{d r^{2}}{\left(2 r^{2} \Lambda / 3+c_{5} / r+\left(c_{2}{ }^{2}+c_{4}{ }^{2}\right) / r^{2}(1-16 \alpha \Lambda)\right)}$

$+r^{2}\left(d \theta_{1}^{2}+d \theta_{2}^{2}\right)$

4.1. Metric with One Variable. From (18) we can deduce the following properties.

(i) In case of 4 dimensions we get

from which it is clear that the metric behaves asymptotically as dS/AdS. Equation (24) shows that the effect of the higher dimension curvature is related to the electric field as well as the magnetic field and also

$d s^{2}$

$$
\Lambda \neq \frac{1}{16 \alpha} .
$$

$=-\left(\frac{2 r^{2} \Lambda}{3}+\frac{c_{5}}{r}+\frac{c_{2}{ }^{2}+c_{4}{ }^{2}}{r^{2}(1-16 \alpha \Lambda)}\right) d t^{2}$

(ii) In case of more than 4 dimensions we get

$$
\begin{aligned}
d s^{2}= & -\frac{(D-2) r^{D-1}\left[1 \pm \sqrt{1-16 D(D-4) \alpha \Lambda /(D-2)^{2}}\right]+2 D(D-1)(D-4) \alpha c_{6}}{2 D(D-1)(D-4) \alpha r^{D-3}} d t^{2} \\
& +\frac{2 D(D-1)(D-4) \alpha r^{D-3} d r^{2}}{(D-2) r^{D-1}\left[1 \pm \sqrt{1-16 D(D-4) \alpha \Lambda /(D-2)^{2}}\right]+2 D(D-1)(D-4) \alpha c_{6}}+r^{2} \sum_{i=1}^{D-2} d \theta^{2}{ }_{i} .
\end{aligned}
$$


Equation (26) shows that the dimensional parameter $\alpha$ must not be equal to zero; otherwise, we will have a singular metric. Also the spacetime of metric (26) behaves as dS/AdS and when the cosmological constant $\Lambda$ takes the form

$$
\Lambda=\frac{(D-2)^{2}}{16 D(D-4) \alpha},
$$

then, (26) reduces to

$$
\begin{aligned}
d s^{2}= & -\frac{(D-2) r^{D-1}+2 D(D-1)(D-4) \alpha c_{6}}{2 D(D-1)(D-4) \alpha r^{D-3}} d t^{2} \\
& +\frac{2 D(D-1)(D-4) \alpha r^{D-3} d r^{2}}{(D-2) r^{D-1}+2 D(D-1)(D-4) \alpha c_{6}} \\
& +r^{2} \sum_{i=1}^{D-2} d \theta^{2}{ }_{i},
\end{aligned}
$$

which is asymptotically $\mathrm{dS} / \mathrm{AdS}$ and cannot reduce to GR.

4.2. Metric with Two Variables. From (23), we can show the following. (iii) In case of 4 dimensions we get

(1) $d s^{2}$

$$
\begin{aligned}
= & -\frac{c_{10}{ }^{2}\left[2 r^{4} \Lambda(16 \alpha \Lambda-1)+48 c_{11} \alpha \Lambda r+3 c_{12}{ }^{2}+3 c_{11} r\right]}{r^{2}} d t^{2} \\
& +\frac{3 r^{2}(16 \alpha \Lambda-1)}{2 r^{4} \Lambda(16 \alpha \Lambda-1)+48 c_{11} \alpha \Lambda r-3 c_{12}{ }^{2}-3 c_{11} r} d r^{2} \\
& +r^{2}\left(d \theta_{1}^{2}+d \theta_{2}^{2}\right) .
\end{aligned}
$$

(2) $d s^{2}$

$$
\begin{aligned}
= & -\frac{c_{15}^{2}\left[2 \Lambda r^{4}+3\left(c_{18}+r c_{19}\right)\right]}{3 r^{2}\left(c_{16}{ }^{2}+c_{18}[16 \alpha \Lambda-1]\right)} d t^{2} \\
& +\frac{3 r^{2}}{3\left(c_{18}+r c_{19}\right)+2 r^{4} \Lambda} d r^{2}+r^{2}\left(d \theta_{1}^{2}+d \theta_{2}^{2}\right) .
\end{aligned}
$$

First set of equations (29) shows that $\Lambda \neq 1 / 16 \alpha$ and when the constant $c_{10}=1 / \sqrt{3(16 \alpha \Lambda-1)}$ then the metric behaves as dS/AdS. For the second set, it is allowed to put $\Lambda=1 / 16 \alpha$ and whenthe constant $c_{15}=c_{16}$ then the second equation of (29) behaves as dS/AdS.

(iv) In case of more than 4 dimensions we get from (23) the two sets

$$
\begin{aligned}
d s^{2}= & -\left\{\frac{(D-2) r^{D-1}\left[\sqrt{1-16 D(D-4) \alpha \Lambda /(D-2)^{2}} \pm 1\right] \pm c_{13}}{r^{D-3}}\right\} d t^{2} \\
& +\left[\frac{(D-2) r^{D-1}\left[\sqrt{1-16 D(D-4) \alpha \Lambda /(D-2)^{2}} \pm 1\right]}{2 D(D-1)(D-4) \alpha r^{D-3}} \pm \frac{c_{13}}{r^{D-3}}\right]^{-1} d r^{2}+r^{2} \sum_{i=1}^{D-2} d \theta^{2}{ }_{i} .
\end{aligned}
$$

Equation (30) shows that the dimensional parameter $\alpha$ must not be equal to zero; otherwise, we will have a singular metric. The asymptotes of (30) behave as dS/AdS. It is important to stress that metric (30) cannot reduce to that of GR and hence we can say that solution (22) is a new solution. Using condition given by (27) in (30) we get

(1) $d s^{2}$

$$
\begin{aligned}
= & -\left\{\frac{ \pm(D-2) r^{D-1} \pm c_{13}}{r^{D-3}}\right\} d t^{2} \\
& +\left[\frac{ \pm(D-2) r^{D-1}}{2 D(D-1)(D-4) \alpha r^{D-3}} \pm \frac{c_{13}}{r^{D-3}}\right]^{-1} d r^{2} \\
& +r^{2} \sum_{i=1}^{D-2} d \theta_{i}^{2} .
\end{aligned}
$$

We can conclude from the above discussion of the metrics given by (30) or (31) that there is no charged solution for the form of $f(R)=R+\alpha R^{2}$. Also spacetime metrics of (30) or (31) instruct us that the dimension parameter $\alpha$ must not be equal zero.

4.3. Singularities. Now, let us explain the singularities and the horizons of solutions (17) and (22). For this reason, we have to find at which values of $r$ do the functions $a(r)$ and $b(r)$ turn out to be zero or infinity, due to the fact that singularities may be coordinate ones which are physical singularities. Usually, to study singularities one calculates all the invariants constructed from Riemann tensor and its contractions. The curvature invariants that arise from solution (17), in case of 4 dimensions, have the form 


$$
\begin{aligned}
R^{\mu \nu \lambda \rho} R_{\mu \nu \lambda \rho} & =\frac{4 r^{2}(1-16 \alpha \Lambda)^{2}\left[8 \Lambda^{2} r^{6}+9 c_{5}{ }^{2}\right]+24\left(c_{2}{ }^{2}+c_{4}{ }^{2}\right)\left[6(1-16 \alpha \Lambda)+7\left(c_{2}{ }^{2}+c_{4}{ }^{2}\right)\right]}{3 r^{8}(1-16 \alpha \Lambda)^{2}}, \\
R^{\mu \nu} R_{\mu \nu} & =\frac{4\left[4 r^{8} \Lambda^{2}(1-16 \alpha \Lambda)^{2}+\left(c_{2}{ }^{2}+c_{4}^{2}\right)^{2}\right]}{r^{8}(1-16 \alpha \Lambda)^{2}}, \quad R=-8 \Lambda .
\end{aligned}
$$

And in case of more than 4 dimensions we get

$$
\begin{aligned}
R^{\mu \nu \lambda \rho} R_{\mu \nu \lambda \rho} & =\frac{r^{4} a^{\prime \prime}+2(D-2) r^{2} a^{2}+2(D-2)(D-3) a^{2}}{r^{4}} \\
R^{\mu \nu} R_{\mu \nu} & =\frac{D(D-2) r^{2} a^{\prime 2}+2(D-2) r^{3} a^{\prime} a^{\prime \prime}+r^{4} a^{\prime \prime}+4(D-2)(D-3) r a a^{\prime}+(D-2)(D-3)^{2} a^{2}}{2 r^{4}} \\
R & =-\frac{2(D-2) r a^{\prime}+r^{2} a^{\prime \prime}+(D-2)(D-3) a}{r^{4}}
\end{aligned}
$$

Equation (32), for the 4 dimensions case, shows the following.

(a) $\Lambda \neq 1 / 16 \alpha$; otherwise, we will have a singularity for both invariants $R^{\mu \nu \lambda \rho} R_{\mu \nu \lambda \rho}$ and $R^{\mu \nu} R_{\mu \nu}$.

(b) Also (32) tells us that there is a singularity at $r=0$ which can not be removed for the invariants $R^{\mu \nu \lambda \rho} R_{\mu \nu \lambda \rho}$ and $R^{\mu \nu} R_{\mu \nu}$.

When $D>4$ and by using (17), we can show the following.

(c) We have a true singularity at $r=0$.

(d) From (33), after using (17), we can show that the dimension parameter $\alpha$ must not be equal to zero; otherwise, we get a singularity.

(e) Finally, (33) shows that there is a singularity at

$$
\begin{aligned}
c_{6} & =-\frac{(D-2) r^{(D-1)}}{2 D(D-1)(D-4) \alpha}[1 \\
& \left. \pm \sqrt{1-\frac{16 D(D-4) \alpha \Lambda}{(D-2)^{2}}}\right],
\end{aligned}
$$

and when constraint (27) is used, (34) takes the form

$$
c_{6}=-\frac{(D-2) r^{(D-1)}}{2 D(D-1)(D-4) \alpha} \text {. }
$$

Repeating the same calculations for solution (22) we get for $D=4$

(1) $R^{\mu \nu \lambda \rho} R_{\mu \nu \lambda \rho}=\frac{4 r^{2}(1-16 \alpha \Lambda)^{2}\left[8 \Lambda^{2} r^{6}+9 c_{11}^{2}\right]+9 c_{11} c_{12}{ }^{2} r(1-16 \alpha \Lambda)+168 c_{11}^{4}}{3 r^{8}(1-16 \alpha \Lambda)^{2}}$

(2) $R^{\mu \nu \lambda \rho} R_{\mu \nu \lambda \rho}=\frac{36 r^{2} c_{19}+144 r c_{19} c_{18}+168 c_{18}{ }^{4}+32 r^{8} \Lambda^{2}}{3 r^{8}}$

(1) $R^{\mu \nu} R_{\mu \nu}=\frac{4\left[c_{12}{ }^{4}-128 r^{8} \Lambda^{3} \alpha(1-8 \alpha \Lambda)+4 r^{8} \Lambda^{2}\right]}{r^{8}(1-16 \alpha \Lambda)^{2}}$,

(2) $R^{\mu \nu} R_{\mu \nu}=\frac{4\left[c_{18}^{2}+4 r^{8} \Lambda^{2}\right]}{r^{8}}$,

(1) $R=-8 \Lambda$

(2) $R=-8 \Lambda$. 
We can apply the same discussion applied for solution (17). In case of $D>4$ we get

$$
\begin{aligned}
& R^{\mu \nu \lambda \rho} R_{\mu \nu \lambda \rho}=\frac{f(r)}{r^{2(D-1)} \alpha^{2}\left((D-2) r^{(D-1)}\left[1 \pm \sqrt{1-16 D(D-4) \alpha \Lambda /(D-2)^{2}}\right] \pm 2 D(D-1)(D-4) \alpha c_{13}\right)^{2}}, \\
& R^{\mu \nu} R_{\mu \nu}=\frac{f_{1}(r)}{\alpha^{2}\left((D-2) r^{(D-1)}\left[1 \pm \sqrt{1-16 D(D-4) \alpha \Lambda /(D-2)^{2}}\right] \pm 2 D(D-1)(D-4) \alpha c_{13}\right)^{2}}, \\
& R=\frac{f_{2}(r)}{\alpha\left((D-2) r^{(D-1)}\left[1 \pm \sqrt{1-16 D(D-4) \alpha \Lambda /(D-2)^{2}}\right] \pm 2 D(D-1)(D-4) \alpha c_{13}\right)},
\end{aligned}
$$

where $f(r), f_{1}(r)$, and $f_{2}(r)$ are lengthy functions of $r$. Using (27) in (37) we get

$$
\begin{aligned}
& R^{\mu \nu \lambda \rho} R_{\mu \nu \lambda \rho} \\
& =\frac{(D-2)^{2}\left[r^{2(D-1)}+2 D(D-1)^{2}(D-3)(D-4)^{2} \alpha^{2} c_{13}^{2}\right]}{2 D(D-1)(D-4)^{2} \alpha^{2}}, \\
& R^{\mu \nu} R_{\mu \nu}=\frac{(D-2)^{2}}{4 D(D-4)^{2} \alpha^{2}}, \\
& R=\frac{-(D-2)}{2(D-4) \alpha},
\end{aligned}
$$

which indicates that the parameter $\alpha$ must not be equal to zero.

The horizons of solutions (17) and (22) are the zeros of the metric $g_{r r}$; therefore, all the above singularities are far from these horizons. The study of perturbations of solutions (17) and (22) is an important issue to study their stability and then discuss the formulation of weak cosmic censorship. This problem will be discussed elsewhere.

\section{Total Conserved Charge}

In this section, we are going to study the conserved quantities of the solutions derived in Section 3. For this purpose we are going to make a brief review of the geometry used in this calculations. The Lagrangian of Einstein-Cartan theory is defined by [100] (the fundamental entities of this theory Appendix A)

$$
S\left(\vartheta^{i}, R_{k}^{j}\right):=-\frac{1}{2 \kappa}\left(R^{i j} \wedge \eta_{i j}-2 \Lambda \eta\right),
$$

where $\vartheta^{i}$ is the one-form coframe and $R^{i j}$ is the two-form curvature tensor. Carrying out variation of (39) with respect to the coframes $\vartheta^{i}$ and $R^{i j}$ lead to $[100,101]$

$$
\begin{aligned}
E_{i} & :=-\frac{\partial S}{\partial 9^{i}}=-\frac{1}{2 \kappa}\left(R^{j k} \wedge \eta_{i j k}-2 \Lambda \eta_{i}\right), \\
B_{i j} & :=-\frac{\partial S}{R_{i j}}=\frac{1}{2 \kappa} \lambda_{i j},
\end{aligned}
$$

which are the canonical energy-momentum and rotational gauge field momentum, respectively. The translational momentum and the spin 2-forms are defined as

$$
\begin{aligned}
& H_{i}:=-\frac{\partial S}{\partial T^{i}}=0, \\
& E_{i j}:=-\vartheta_{[i} \wedge H_{j]}=0 .
\end{aligned}
$$

The conserved quantity of the gravitational field has the form [100]

$$
\begin{aligned}
&\left.J[\xi]=\frac{1}{2 \kappa} d\left\{{ }^{*}[d k+\xi\rfloor\left(\vartheta^{i} \wedge T_{i}\right)\right]\right\}, \\
&\text { where } \left.k=\xi_{i} \vartheta^{i}, \xi^{i}=\xi\right\rfloor \vartheta^{i},
\end{aligned}
$$

with $*$ being the Hodge duality, $\xi$ being a vector field $\xi=$ $\xi^{i} \partial_{i}$, and $\xi^{i}$ being $D$ parameters $\xi^{0}, \xi^{1}, \ldots, \xi^{D-1}$. For the solutions having spinless matter or vacuum ones, the torsion is vanishing, $T_{i}$, and therefore the total charge of (42) takes the form

$$
\mathcal{Q}[\xi]=\frac{1}{2 \kappa} \int_{\partial S}^{*} d k
$$

This invariant conserved quantity $\mathcal{Q}[\xi]$ was given before in [102-106].

Now let us apply (43) to solution (17) and calculate the necessary components; we finally get

$$
\begin{gathered}
\vartheta^{0}=\sqrt{a(r)} d t, \\
\vartheta^{1}=\frac{d r}{\sqrt{a(r)}}, \\
\vartheta^{2}=r d \theta_{1}, \\
\vartheta^{3}=r d \theta_{2}, \\
\vdots \\
\vartheta^{D-1}=r d \theta_{D-2} .
\end{gathered}
$$




$$
k=\frac{a^{2}(r) \xi_{0} d t-\xi_{1} d r-r^{2} a(r)\left(\xi_{2} d \theta_{2}+\xi_{3} d \theta_{2}+\cdots+\xi_{D-2} d \theta_{D-2}\right)}{a(r)} .
$$

The total derivative of (45) gives

$$
\begin{aligned}
d k & =\xi_{0} a^{\prime}(r)(d r \wedge d t)-2 r\left[\xi_{2}\left(d r \wedge d \theta_{1}\right)\right. \\
& \left.+\xi_{3}\left(d r \wedge d \theta_{2}\right)+\cdots+\xi_{D-1}\left(d r \wedge d \theta_{D-2}\right)\right] .
\end{aligned}
$$

Calculating the inverse of (46) and using it in (46) and (43) after applying the Hodge-dual to the output of $d k$, we get conservation of the charge in the form

$$
\begin{aligned}
& \mathbb{Q}\left[\xi_{t}\right]=\frac{(D-2) \Omega_{D-2} M}{8 \pi}, \\
& \mathbb{Q}\left[\xi_{r}\right]=\mathscr{Q}\left[\xi_{\theta_{1}}\right]=\cdots=\mathscr{Q}\left[\xi_{\theta_{D-2}}\right]=0,
\end{aligned}
$$

where $\Omega_{D-2}$ is the volume of the unit $(D-2)$-sphere; we have substitute $c_{5}=-2 M$ and have used the regularization method, by putting the physical quantities vanishing, and subtract the resulting expression from the original one to remove any divergence term [100] (physical quantities mean the constants $c_{2}, c_{4}, c_{5}$, and $c_{6}$ for solution (17); constants 10 , 11,12 , and 13 of first solution of (22); and constants 13, 15, 18, and $c_{18}$ of the second solution of (22)).

Applying the same above techniques we get for the first solution of (22) in case of $D=4$

$$
\begin{aligned}
& \mathbb{Q}\left[\xi_{t}\right]=M \sqrt{1-16 \alpha \Lambda}, \\
& \mathbb{Q}\left[\xi_{r}\right]=\mathscr{Q}\left[\xi_{\theta_{1}}\right]=\cdots=\mathscr{Q}\left[\xi_{\theta_{D-2}}\right]=0,
\end{aligned}
$$

where $c_{11}=-2 M / \sqrt{c_{10}}$ and for the second one we get

$$
\begin{aligned}
& \mathbb{Q}\left[\xi_{t}\right]=\frac{M}{\sqrt{c_{18}(1-16 \alpha \Lambda)-c_{17}^{2}}}, \\
& \mathbb{Q}\left[\xi_{r}\right]=\mathbb{Q}\left[\xi_{\theta_{1}}\right]=\cdots=\mathbb{Q}\left[\xi_{\theta_{D-2}}\right]=0,
\end{aligned}
$$

where $c_{19}=-2 M / c_{15}$.

In case $D>4$ we get

$$
\begin{aligned}
& \mathbb{Q}\left[\xi_{t}\right]=\frac{(D-2) \Omega_{D-2} M \sqrt{\alpha}}{8 \pi}, \\
& \mathbb{Q}\left[\xi_{r}\right]=\mathscr{Q}\left[\xi_{\theta_{1}}\right]=\cdots=\mathscr{Q}\left[\xi_{\theta_{D-2}}\right]=0,
\end{aligned}
$$

where

$$
c_{13}=-\frac{2 M \sqrt{\alpha D(D-3)(D-4)}}{\sqrt{c_{15}}} .
$$

\section{Thermodynamics of Black Holes}

In this section, we are going to study the thermodynamical quantities of solutions (17) and (22). The temperature of
Hawking of any solution can be derived by requiring the singularity at the horizon to be vanishing in the Euclidean continuation of the black hole solutions. One can obtain the temperature of the outer event horizon at $r=r_{h}$, for solution (17) in case of $D=4$ in the form

$$
\begin{aligned}
T & =\frac{1}{4 \pi}\left(\frac{d g_{t t}(\mathrm{r})}{d r}\right)_{r_{h}} \\
& =\frac{1}{4 \pi}\left[2 r_{h} \Lambda+\frac{c_{2}^{2}+c_{4}^{2}}{(16 \alpha \Lambda-1) r_{h}^{3}}\right],
\end{aligned}
$$

and in case of $D>4$ we get

$$
\begin{aligned}
T & =\frac{1}{4 \pi}\left(\frac{d g_{t t}(r)}{d r}\right)_{r_{h}} \\
& =\frac{r_{h}}{4 \pi}\left[\frac{(D-2)\left[1 \pm \sqrt{1-16 D(D-4) \alpha \Lambda /(D-2)^{2}}\right]}{2 D(D-4) \alpha}\right] .
\end{aligned}
$$

For the first solution of (22) in case $D=4$, we get the Hawking temperature as

$$
\begin{aligned}
T & =\frac{1}{4 \pi}\left(\frac{d g_{t t}(r)}{d r}\right)_{r_{h}} \\
& =\frac{1}{4 \pi}\left[\frac{3 c_{10}\left[2 \Lambda r^{4}{ }_{h}(1-16 \alpha \Lambda)+c_{11}{ }^{2}\right]}{r^{3}}\right],
\end{aligned}
$$

and for the second solution of (22) in case of $D=4$, we get

$$
\begin{aligned}
T & =\frac{1}{4 \pi}\left(\frac{d g_{t t}(r)}{d r}\right)_{r_{h}} \\
& =\frac{1}{4 \pi}\left[\frac{c_{15}{ }^{2}\left[\left(c_{18}-2 \Lambda r^{4}{ }_{h}\right)\right]}{r^{3}{ }_{h}\left[c_{18}(16 \alpha \Lambda-1)+c_{16}^{2}\right]}\right] .
\end{aligned}
$$

In the case of $D>4$ we get

$$
\begin{aligned}
T & =\frac{1}{4 \pi}\left(\frac{d g_{t t}(r)}{d r}\right)_{r_{h}} \\
& =\frac{r_{h} c_{13}(D-1)(D-2)}{4 \pi}\left[\sqrt{1-\frac{16 D(D-4) \alpha \Lambda}{(D-2)^{2}}}\right. \\
& \pm 1] .
\end{aligned}
$$

We give a brief discussion of the entropy of black hole in $f(R)$ gravity. For this purpose, we use the arguments presented in [107]. From the Noether method used to calculate the entropy 
associated with black holes in $f(R)$ theory that have constant Ricci scalar, one finds [95]

$$
S=\left.\frac{A}{4 G} f^{\prime}(R)\right|_{r=r_{h}}
$$

where $A$ is the area of the event horizon. Using solution (17) we get for $D=4$

$$
S=(1-16 \Lambda \alpha) \pi r_{h}^{2},
$$

and for $D>4$ we get

$$
\begin{aligned}
S= & \frac{r_{h}{ }^{D-2} \Omega_{D-2}}{4}[1 \\
& \left.-\frac{(D-2)}{(D-4)}\left\{1+\sqrt{1-\frac{16 D(D-4) \alpha \Lambda}{(D-2)^{2}}}\right\}\right] .
\end{aligned}
$$

Using (17) and (27) in (59) we get the entropy in the form

$$
S=\frac{r_{h}{ }^{D-2} \Omega_{D-2}}{2(D-4)}
$$

For solution (22) and in case of $D=4$ we get the same expression of entropy as given by (58) and in case $D>4$ we get

$$
S=\frac{r_{h}{ }^{D-2} \Omega_{D-2}}{4}\left\{1+2 \alpha R\left(r_{h}\right)\right\} .
$$

When (27) is used we get the entropy in the form of (60).
Utilizing (52) and (58), the Smarr relation in the extended phase space can be obtained in the case $D=4$ of solution (17) as

$$
\begin{aligned}
& M=2 T S+\Phi Q, \\
& \text { where } \Phi=\sqrt{\frac{\left(c_{2}{ }^{2}+c_{4}{ }^{2}\right)(1-\Lambda \alpha)}{r_{h}}}, Q=\sqrt{\left(c_{2}{ }^{2}+c_{4}{ }^{2}\right)(1-\Lambda \alpha)} .
\end{aligned}
$$

For the first solution of (22) we get the the Smarr relation in the extended phase as

$$
\begin{aligned}
& M=2 T S+\Phi Q, \\
& \text { where } \Phi=\frac{c_{11}}{r \sqrt{(1-2 \Lambda \alpha)}}, Q=\frac{c_{11}}{\sqrt{1-2 \Lambda \alpha}},
\end{aligned}
$$

where we have put $c_{10}=1 / 3(1-2 \Lambda \alpha)^{2}$. Finally, for the second solution of (22) we get the Smarr relation as

$$
M=2 T S+\Phi Q, \quad \text { where } \Phi=\frac{\sqrt{2 c_{18}}}{r_{h}}, Q=\sqrt{2 c_{18}},
$$

where we have put $c_{15}=\sqrt{\left(c_{18}(1-16 \alpha \Lambda)-c_{16}^{2}\right) /(16 \alpha \Lambda-1)}$.

In the case $D>4$, we introduce the extended phase space where the cosmological constant is identified as the thermodynamic pressure while the conjugate quantity is regarded as the thermodynamic volume. We adopt the following definition of pressure that is commonly used in the literatures of extended phase space [108]

$$
P=-\frac{(D-1)(D-2) \Lambda}{48 \pi} .
$$

Using solution (17) in the case $D>4$ we get the Smarr relation in the extended phase as [108]

$$
\begin{aligned}
& M=\frac{D-2}{D-3} T S-\frac{2}{D-3} V P, \text { where } P \text { is given by Eq. (65) and } V \text { has the form } \\
& V=\frac{3(D-2) r_{h}{ }^{D-1} \Omega_{D-2}\left(2+(D-2) \sqrt{1-16 D(D-4) \alpha \Lambda /(D-2)^{2}}\right)\left(1+\sqrt{1-16 D(D-4) \alpha \Lambda /(D-2)^{2}}\right)}{4 \alpha \Lambda D(D-1)(D-4)^{2}} .
\end{aligned}
$$

It is important to note that Smarr relation given by (66) has no charge term because the higher dimension solution given by Eq. (17) has constant electric and magnetic charges. Using constrains (27) in (66) we get

$$
V=\frac{24 r_{h}{ }^{D-1} \Omega_{D-2}}{(D-1)(D-2)(D-4)} .
$$

Using the same above procedure we get for solution (22) in case of $D>4$ the the Smarr relation in the extended phase as given by (66) where the volume has the form

$$
V=\frac{2 r_{h}{ }^{D-1} \Omega_{D-2}}{(D-4)}
$$

\section{Main Results and Discussion}

In this study, we have presented Maxwell- $f(R)$ gravity in $D$-dimensions and have checked the flat horizon solutions. We have applied two metrics, the first with one unknown and the second with two unknown functions to Maxwell$f(R)$ field equations using the special case $f(R)=R+\alpha R^{2}$. The resulting differential equations are solved analytically without any assumption and general solutions containing 
three classes have been obtained. These classes are classified as follows.

(i) For the metric with one unknown, the electric and magnetic charges of this class are not constant for $D=4$ and are constants for $D>4$.

(ii) For the metric with two unknowns, we have two sets. The first one has a constant charge and a nontrivial magnetic field for $D=4$ and for $D>4$ we have constant electric and magnetic charges.

(iii) For the second set of solutions (22) and for $D=4$ the electric and magnetic charges are not trivial and are constants for $D>4$.

To understand the physics of these solutions we wrote the metric of each solution and have shown the following.

(a) We have shown that the metric behaves asymptotically as dS/AdS for the first solution for $D=4$ and it is not allowed to put $\Lambda=1 / 16 \alpha$. When $D>4$ the metric also behaves asymptotically as dS/AdS and have shown that the following relation between the cosmological constant and the dimension parameter $\alpha$ holds $\Lambda=(D-2)^{2} / 16 D(D-4) \alpha$.

(b) For the metric with two unknowns, when $D=4$, we have shown that the metric behaves as $\mathrm{dS} / \mathrm{Ads}$ for both sets. However, for the first solution $\Lambda \neq 1 / 16 \alpha$ but for the second one $\Lambda=1 / 16 \alpha$.

(c) When $D>4$, for solution (22), we have shown that the parameter $\alpha$ can not be equal to zero and therefore this solution is new.

We have calculated the singularities of each class by calculating the invariants of curvature. For the first solution we have shown that the invariants of curvature have singularity at $r=0$ and $\Lambda=1 / 16 \alpha$. However, when $D>4$ we have shown that there is singularities at $r=0$ and $\alpha=0$. For the first set of (22) the same discussion of solution (17) can be applied for the invariants of curvature. However, for the second set the invariants of curvature have a singularity at $r=0$ which represents horizon. For $D>4$ the same discussion of solution (17) can be applied for the two sets.

To understand the meaning of the constants of each solution we have calculated the conserved quantities. In the case of $D=4$ we have shown that the only nonvanishing physical quantity is the energy. We have shown that the value of energy of solution (17) is not affected by the dimension parameter $\alpha$ while it is affected for solution (22) and reduces to the well known formula of ADM when $\alpha=0$. When $D>4$ the energy of solution (17) does not depend on the parameter $\alpha$ while it depends on it for solution (22) and can not be equal to zero.

Finally, we have calculated the thermodynamical quantities like Hawking temperature, entropy, and so on. For solution (17) and when $D=4$ we have shown that the temperature depends on the electric and magnetic charges and for $D>4$ the parameter $\alpha$ is not allowed to be zero; otherwise, the temperature will be indefinite. We have shown that the entropy, for solution (17), depends on $\alpha$ and when it is vanishing we return to GR. For this solution, we have shown that the first law of thermodynamics is satisfied for general $D \geq 4$ using the extended phase space [108].

For solution (22) we have shown that temperature will be finite if $\alpha=0$ and will return to GR after rescaling the constants of integration. For the entropy and when $D=4$ the same discussion of solution (17) can be applied. The entropy will have a finite value only when the constraint (27) is used. Finally we have shown that the first law of thermodynamics is satisfied for general $D \geq 4$.

\section{Appendix}

\section{A. Notation}

The indices $i, j, \ldots$ are employed for (co)frame components while $\alpha, \beta, \ldots$ are used for spacetime coordinates. The exterior product is represented by $\wedge$ and the interior product is denoted by $\xi\rfloor \Psi$. The coframe $\vartheta^{i}$ is defined as $\vartheta^{i}=b_{\mu}^{i} d x^{\mu}$ and the frame $b_{i}$ is defined as $b_{i}=b_{i}{ }^{\mu} \partial_{\mu}$ with $b_{\mu}{ }_{\mu}$ and $b_{i}{ }^{\mu}$ being the covariant and contravariant components of the tetrad field. The volume is defined as $\eta:=\vartheta^{\widehat{0}} \wedge \vartheta^{\hat{1}} \wedge \vartheta^{\widehat{2}} \wedge \vartheta^{\hat{3}}$. Using the interior product one can define

$$
\left.\eta_{i}:=b_{i}\right\rfloor \eta=\frac{1}{3 !} \epsilon_{i j k l} \vartheta^{j} \wedge \vartheta^{k} \wedge \vartheta^{l},
$$

with $\epsilon_{i j k l}$ being completely antisymmetric.

\section{B. The Nonvanishing Components of the Christoffel Symbols Second Kind and Ricci Curvature Tensor}

Using (13) we get the following nonvanishing components of the Christoffel symbols second kind and Ricci curvature tensor:

$$
\begin{aligned}
& \Gamma_{t t}^{t}=-\Gamma_{r r}^{r}=\frac{a^{\prime}}{2 a}, \\
& \Gamma_{r t}^{t}=\frac{a a^{\prime}}{2}, \\
& \Gamma_{r \theta_{1}}^{\theta_{1}}=\Gamma_{r \theta_{2}}^{\theta_{2}} \cdots \Gamma^{\theta_{D-2}}{ }_{r \theta_{D-2}}=\frac{1}{r}, \\
& \Gamma_{\theta_{1} \theta_{1}}^{r}=\Gamma^{r}{ }_{\theta_{2} \theta_{2}} \cdots \Gamma_{\theta_{D-2} \theta_{D-2}}^{r}=-r a . \\
& R_{t r t r}=\frac{a^{\prime \prime}}{2}, \\
& R_{t \theta_{1} t \theta_{1}}=R_{t \theta_{2} t \theta_{2}}=\cdots=R_{t \theta_{D-2} t \theta_{D-2}}=\frac{r a a^{\prime}}{2}, \\
& R_{r \theta_{1} r \theta_{1}}=R_{r \theta_{2} r \theta_{2}}=\cdots=R_{r \theta_{D-2} r \theta_{D-2}}=-\frac{r a^{\prime}}{2 a}, \\
& R_{\theta_{1} \theta_{2} \theta_{1} \theta_{2}}=R_{\theta_{1} \theta_{2} \theta_{1} \theta_{2}}=\cdots=R_{\theta_{1} \theta_{D-2} \theta_{1} \theta_{D-2}}=R_{\theta_{2} \theta_{3} \theta_{2} \theta_{3}} \\
& \quad=R_{\theta_{2} \theta_{4} \theta_{2} \theta_{4}} \cdots R_{\theta_{2} \theta_{D-2} \theta_{2} \theta_{D-2}} \cdots R_{\theta_{D-3} \theta_{D-2} \theta_{D-3} \theta_{D-2}} \\
& =-r^{2} a .
\end{aligned}
$$


Using (19) we get the following nonvanishing components of the Christoffel symbols second kind and Ricci curvature tensor:

$$
\begin{aligned}
& \Gamma_{t t}^{t}=\frac{a^{\prime}}{2 a} \\
& \Gamma_{r r}^{r}=\frac{b^{\prime}}{2 b}, \\
& \Gamma_{r t}^{t}=\frac{a a^{\prime}}{2}, \\
& \Gamma_{r \theta_{1}}^{\theta_{1}}=\Gamma^{\theta_{2}}{ }_{r \theta_{2}} \cdots \Gamma^{\theta_{D-2}} r \theta_{D-2}=\frac{1}{r}, \\
& \Gamma_{\theta_{1} \theta_{1}}^{r}=\Gamma^{r}{ }_{\theta_{2} \theta_{2}} \cdots \Gamma^{r} \theta_{D-2} \theta_{D-2}=-r b . \\
& R_{t r t r}=\frac{a^{\prime \prime}}{2}-\frac{a^{\prime 2}}{4 a}+\frac{a^{\prime} b^{\prime}}{4 b}, \\
& R_{t \theta_{1} t \theta_{1}}=R_{t \theta_{2} t \theta_{2}}=\cdots=R_{t \theta_{D-2} t \theta_{D-2}}=\frac{r b a^{\prime}}{2}, \\
& R_{r \theta_{1} r \theta_{1}}=R_{r \theta_{2} r \theta_{2}}=\cdots=R_{r \theta_{D-2} r \theta_{D-2}}=-\frac{r b^{\prime}}{2 b}, \\
& R_{\theta_{1} \theta_{2} \theta_{1} \theta_{2}}=R_{\theta_{1} \theta_{2} \theta_{1} \theta_{2}}=\cdots=R_{\theta_{1} \theta_{D-2} \theta_{1} \theta_{D-2}}=R_{\theta_{2} \theta_{3} \theta_{2} \theta_{3}} \\
& =R_{\theta_{2} \theta_{4} \theta_{2} \theta_{4}} \cdots R_{\theta_{2} \theta_{D-2} \theta_{2} \theta_{D-2}} \cdots R_{\theta_{D-3} \theta_{D-2} \theta_{D-3} \theta_{D-2}} \\
& =-r^{2} b .
\end{aligned}
$$

\section{Conflicts of Interest}

The author declares that there are no conflicts of interest regarding the publication of this paper.

\section{References}

[1] A. G. Riess and etal., "Supernova search team collaboration," The Astronomical Journal, vol. 116, p. 1009, 1998.

[2] S. Perlmutter, G. Aldering, and G. Goldhaber, "Measurements of omega and lambda from 42 high-redshift supernovae," The Astrophysical Journal, vol. 517, no. 2, p. 565, 1999.

[3] J. L. Tonry and etal., "Cosmological results from high-z supernovae," The Astrophysical Journal, vol. 594, pp. 1-24, 2003.

[4] A. H. Guth, "Inflationary universe: A possible solution to the horizon and flatness problems," Phys. Rev. D, vol. 23, pp. 347356, 1981.

[5] P. J. E. Peebles, Principles of Physical Cosmology, Princeton University Press, Princeton, NJ, USA, 1993.

[6] E. Komatsu and etal., "Seven-Year Wilkinson Microwave Anisotropy Probe (WMAP) Observations: Cosmological Interpretation," The Astrophysical Journal Supplement Series, vol. 2011, no. 192, p. 18, 2011.

[7] W. J. Percival and etal., "Baryon acoustic oscillations in the Sloan Digital Sky Survey Data Release 7 galaxy sample," Mon. Not. Roy. Astron. Soc, vol. 401, pp. 2148-2168, 2010.

[8] A. G. Riess, The Astrophysical Journal, vol. 699, p. 539, 2009.

[9] L. Covi, J. E. Kim, and L. Roszkowski, "Axinos as cold dark matter," Physical Review Letters, vol. 82, no. 21, pp. 4180-4183, 1999.
[10] J. L. Feng, A. Rajaraman, and F. Takayama, "Graviton cosmology in universal extra dimensions," Physical Review D: Particles, Fields, Gravitation and Cosmology, vol. 2003, no. D 68, Article ID 085018, 2003.

[11] J. L. Feng, A. Rajaraman, and F. Takayama, "Probing gravitational interactions of elementary particles," International Journal of Modern Physics D, vol. 13, no. 10, pp. 2355-2359, 2004.

[12] J. A. R. Cembranos, A. de la Cruz-Dombriz, and P. J. Romero, "Kerr-Newman black holes in $\mathrm{f}(\mathrm{R})$ theories," International Journal of Geometric Methods in Modern Physics, vol. 2014, no. 11, Article ID 1450001, 2014.

[13] A. de la Cruz-Dombriz and D. Sáez-Gómez, "Black holes, cosmological solutions, future singularities, and their thermodynamical properties in modified gravity theories," Entropy, vol. 2012, no. 14, pp. 1717-1770, 2012.

[14] S. H. Hendi and D. Momeni, "Black-hole solutions in $F(R)$ gravity with conformal anomaly," The European Physical Journal C, vol. 71, no. 12, article 1823, 2011.

[15] J. A. R. Cembranos, J. L. Feng, A. Rajaraman, and F. Takayama, "Superweakly Interacting Massive Particle Solutions to Small Scale Structure Problems," Physical Review Letters, vol. 95, no. 18, 2005.

[16] F. D. Albareti, J. A. R. Cembranos, and A. de la Cruz-Dombriz, "Focusing of geodesic congruences in an accelerated expanding Universe," Journal of Cosmology and Astroparticle Physics, vol. 2012, no. 12, pp. 020-020, 2012.

[17] J. A. R. Cembranos, J. L. Feng, and L. E. Strigari, "Exotic collider signals from the complete phase diagram of minimal universal extra dimensions," Physical Review D: Particles, Fields, Gravitation and Cosmology, vol. 2007, no. D 75, Article ID 036004, 2007.

[18] J. A. R. Cembranos, J. H. Montes de, Y. Oca, and L. Prado, "Dark Matter and Higgs Sector," J. Phys. Conf. Ser, vol. 315, Article ID 012012, 2011.

[19] H. Goldberg, "Constraint on the photino mass from cosmology," Physical Review Letters, vol. 50, no. 19, pp. 1419-1422, 1983.

[20] J. R. Ellis and etal., "Supersymmetric relics from the big bang," Nuclear Physics B, vol. 238, no. 2, p. 453, 1984.

[21] K. Griest and M. Kamionkowski, "Supersymmetric Dark Matter," Phys. Rept, vol. 267, p. 195, 1996.

[22] J. A. R. Cembranos, A. Dobado, and A. L. Maroto, "BraneWorld Dark Matter," Physical Review Letters, vol. 2003, no. D 90, Article ID 241301, 2003.

[23] J. A. R. Cembranos, A. Dobado, and A. L. Maroto, "BraneWorld Dark Matter," Physical Review Letters, vol. 2003, no. D 68, Article ID 103505, 2003.

[24] J. A. R. Cembranos, A. Dobado, and A. L. Maroto, "Phenomenological implications of brane world scenarios with low tension," AIP Conf. Proc, vol. 670, pp. 235-242, 2003.

[25] J. A. R. Cembranos, "The Newtonian limit at intermediate energies," Physical Review D: Particles, Fields, Gravitation and Cosmology, vol. 2006, no. D 73, Article ID 035008, 2006.

[26] J. A. R. Cembranos, A. Dobado, and A. L. and Maroto, "The Newtonian limit at intermediate energies," Physical Review D: Particles, Fields, Gravitation and Cosmology, vol. D 73, no. 6, Article ID 057303, 2006.

[27] A. L. Maroto, Physical Review D: Particles, Fields, Gravitation and Cosmology, vol. 2004, no. D 69, Article ID 043509, 2004.

[28] A. L. Maroto and D. Phys. Rev, Physical Review D: Particles, Fields, Gravitation and Cosmology, Article ID 101304, 2004. 
[29] A. Dobado and A. L. Maroto, "The dynamics of the Goldstone bosons on the brane," Nuclear Physics B, vol. 592, no. 1-2, pp. 203-218, 2000.

[30] A. Dobado and A. L. Maroto, "Dark Geometry," Int. J. Mod. Phys. D, vol. 13, pp. 2275-2280, 2004.

[31] J. A. R. Cembranos, A. de la Cruz-Dombriz, A. Dobado, and A. L. Maroto, "Is the CMB cold spot a gate to extra dimensions?" Journal of Cosmology and Astroparticle Physics, vol. 2008, Article ID 039, 2008.

[32] J. A. R. Cembranos and L. E. Strigari, "Diffuse $\mathrm{MeV}$ gamma rays and galactic $511 \mathrm{keV}$ line from decaying WIMP dark matter," Physical Review D: Particles, Fields, Gravitation and Cosmology, vol. 2008, no. D 77, Article ID 123519, 2008.

[33] J. A. Cembranos, J. L. Feng, and L. E. Strigari, "Resolving cosmic gamma ray anomalies with dark matter decaying now," Physical Review Letters, vol. 2007, no. 99, Article ID 191301, 2007.

[34] J. A. R. Cembranos, A. de la Cruz-Dombriz, A. Dobado, R. A. Lineros, and A. L. Maroto, "Photon spectra from WIMP annihilation," Physical Review D: Particles, Fields, Gravitation and Cosmology, vol. 83, no. 8, Article ID 083507, 2011.

[35] J. Alcaraz, Physical Review D: Particles, Fields, Gravitation and Cosmology, vol. 2003, no. D67, Article ID 075010, 2003.

[36] P. Achard and etal., "Measurement of exclusive $\rho+\rho$-production in high-Q2 two-photon collisions at LEP," Physics Letters B, vol. 597, no. 1, p. 26, 2004.

[37] P. Achard and etal., "Searching for CPT violation in $t \bar{t}$ production,” EPL (Europhysics Letters), vol. 82, Article ID 21001, 2008.

[38] J. A. R. Cembranos, A. Dobado, and A. L. Maroto, "BraneSkyrmions and wrapped states," Physical Review D: Particles, Fields, Gravitation and Cosmology, vol. 2002, no. D 65, Article ID 026005, 2002.

[39] J. A. Cembranos, A. Dobado, and A. L. Maroto, "Some model-independent phenomenological consequences of flexible braneworlds," Journal of Physics A: Mathematical and General, vol. 40, no. 25, pp. 6631-6640, 2007.

[40] J. A. R. Cembranos, A. Dobado, and A. L. Maroto, "Branon search in hadronic colliders," Physical Review D: Particles, Fields, Gravitation and Cosmology, vol. 2004, no. D 70, Article ID 096001, 2004.

[41] J. A. R. Cembranos, A. de la Cruz-Dombriz, and P. Romero, "Kerr-Newman black holes in $f(R)$ theories," International Journal of Geometric Methods in Modern Physics, vol. 2014, no. 11, Article ID 1450001, 2014.

[42] J. A. R. Cembranos, “Gravitino and Axino SuperWIMPs," AIP Conf. Proc, vol. 903, pp. 591-594, 2007.

[43] T. Biswas and etal., "Erratum: particle accelerators inside spinning black holes," Physical Review Letters, vol. 2010, no. 104, Article ID 021601, 2010.

[44] T. Biswas, "Thermodynamics and Cosmological Constant of Non-Local Field Theories from p-Adic Strings," Journal of High Energy Physics, vol. 2010, no. 1010, p. 048, 2010.

[45] T. Biswas, "Finite Temperature Solitons in Non-Local Field Theories from p-Adic Strings," Physical Review D: Particles, Fields, Gravitation and Cosmology, vol. D 82, Article ID 085028, 2010.

[46] S. Weinberg, "The cosmological constant problem," Reviews of Modern Physics, vol. 61, no. 1, pp. 1-23, 1989.

[47] X. Meng and P. Wang, "Palatini formulation of modified gravity with squared scalar curvature," General Relativity and Gravitation, vol. 36, no. 12, pp. 2673-2680, 2004.
[48] S. Nojiri and S. D. Odintsov, "Modified gravity with negative and positive powers of curvature: Unification of inflation and cosmic acceleration," Physical Review D: Particles, Fields, Gravitation and Cosmology, vol. 68, Article ID 123512, 2003.

[49] S. Nojiri and S. D. Odintsov, "Modified gravity with $\ln R$ terms and cosmic acceleration," General Relativity and Gravitation, vol. 36, no. 8, pp. 1765-1780, 2004.

[50] S. M. Carroll, V. Duvvuri, M. Trodden, and M. S. Turner, "Is cosmic speed-up due to new gravitational physics?" Physical Review D: Particles, Fields, Gravitation and Cosmology, vol. 70, Article ID 043528, 2004.

[51] A. Dobado and A. L. Maroto, "Inflatonless inflation," Physical Review D: Particles, Fields, Gravitation and Cosmology, vol. 1995, no. D 52, pp. 1895-1901, 1995.

[52] G. Dvali, G. Gabadadze, and M. Porrati, “4D gravity on a brane in 5D Minkowski space," Physics Letters B, vol. 2000, no. B 485, p. 208, 2000.

[53] J. A. R. Cembranos, "Dark matter from $R^{2}$ gravity," Physical Review Letters, vol. 2009, no. 102, p. 141301, 2009.

[54] J. A. R. Cembranos, "QCD Effects in Cosmology", AIP Conf. Proc, vol. 1182, pp. 288-291, 2009.

[55] J. A. R. Cembranos, "R ${ }^{2}$ Dark Matter," J. Phys. Conf. Ser, vol. 315, Article ID 012004, 2011.

[56] J. A. Cembranos, "The Newtonian limit at intermediate energies," Physical Review D: Particles, Fields, Gravitation and Cosmology, vol. 2006, no. D 73, p. 064029, 2006.

[57] J. A. R. Cembranos, K. A. Olive, M. Peloso, and J.-P. Uzan, "Quantum corrections to the cosmological evolution of conformally coupled fields," Journal of Cosmology and Astroparticle Physics, vol. 907, article 025, 2009.

[58] S. Nojiri and S. D. Odintsov, "Introduction to modified gravity and gravitational alternative for dark energy," International Journal of Geometric Methods in Modern Physics, vol. 4, no. 1, article 115, 2007.

[59] J. B. Jiménez and A. L. Maroto, "Cosmic vector for dark energy," Physical Review D: Particles, Fields, Gravitation and Cosmology, vol. 2008, no. D 78, Article ID 063005, 2008.

[60] J. Beltran Jimenez and L. Antonio, "Cosmological electromagnetic fields and dark energy," Journal of Cosmology and Astroparticle Physics, vol. 2009, no. 0903, p. 0903, 2009.

[61] J. Beltrán Jiménez and A. L. Maroto, "Cosmological evolution in vector-tensor theories of gravity," Physical Review D: Particles, Fields, Gravitation and Cosmology, vol. 2009, no. D 80, Article ID 063512, 2009.

[62] J. Beltran and A. L. Maroto, "Dark energy: the absolute electric potential of the universe," Int. J. Mod. Phys. D, vol. 18, pp. $2243-$ 2248, 2009.

[63] G. Kofinas, E. Papantonopoulos, and E. N. Saridakis, "Modified Brans-Dicke cosmology with matter-scalar field interaction," Classical and Quantum Gravity, vol. 2016, no. 33, Article ID 155004, 2016.

[64] R. Kallosh, F. Quevedo, and A. M. Uranga, "String theory realizations of the nilpotent goldstino," Journal of High Energy Physics, vol. 2015, no. 1512, p. 39, 2015.

[65] S. H. Hendi and A. Dehghani, "Thermodynamics of thirdorder Lovelock-AdS black holes in the presence of Born-Infeld type nonlinear electrodynamics," Physical Review D: Particles, Fields, Gravitation and Cosmology, vol. 2015, no. D 91, Article ID 064045, 2015.

[66] G. G. L. Nashed, "A spherically-symmetric charged-dS solution in $\mathrm{f}(\mathrm{T})$ gravity theories," Physical Review D: Particles, Fields, Gravitation and Cosmology, vol. D 88, Article ID 104034, 2013. 
67] A. M. Awad, S. Capozziello, and G. G. L. Nashed, "D-dimensional charged Anti-de-Sitter black holes in $f(T)$ gravity," Journal of High Energy Physics, vol. 136, 2017.

[68] G. G. L. Nashed, "Vacuum non singular black hole solutions in tetrad theory of gravitation," General Relativity and Gravitation, vol. 34, no. 7, pp. 1047-1058, 2002.

[69] L. Pogosian and A. Silvestri, "Erratum: Pattern of growth in viable $f(R)$ cosmologies," Physical Review D: Particles, Fields, Gravitation and Cosmology, vol. 2008, no. D 77, Article ID 023503, 2008.

[70] G. G. L. Nashed, Nuovo Cimento B, vol. 2002, no. 117, p. 521, 2002.

[71] G. G. L. Nashed, "Kerr-Newman solution in modified teleparallel theory of gravity," Int. J. Mod. Phys. D, vol. 2015, Article ID 15500071, 2015.

[72] G. G. L. Nashed and W. EL Hanafy, "A built-in inflation in the $\mathrm{f}(\mathrm{T})$-cosmology," The European Physical Journal C, vol. 2014, no. C 74, p. 3099, 2014.

[73] S. Nojiri and S. D. Odintsov, "Unified cosmic history in modified gravity: from $f(R)$ theory to Lorentz non-invariant models," Physics Reports, vol. 2011, p. 59, 2011.

[74] G. G. L. Nashed, "Monte Carlo evaluation of the external gamma, neutron and muon induced background sources in the CUORE experiment," Astroparticle Space Physics, vol. 2010, no. 330, p. 173, 2010.

[75] G. G. L. Nashed, "Spherically Symmetric Solutions on a NonTrivial Frame in $\mathrm{f}(\mathrm{T})$ Theories of Gravity," Chin. Phys. Lett, vol. 2011, no. 29, Article ID 050402, 2011.

[76] D. Saez-Gomez, C. S. Carvalho, F. S. N. Lobo, and I. Tereno, "Constraining $\mathrm{f}(\mathrm{T}, \mathrm{CalT})$ gravity models using type Ia supernovae," Physical Review D: Particles, Fields, Gravitation and Cosmology, vol. 2016, no. D94, Article ID 024034, 2016.

[77] A. Awad and G. Nashed, "Generalized teleparallel cosmology and initial singularity crossing," Journal of Cosmology and Astroparticle Physics, vol. 2017, no. 02, pp. 046-046, 2017.

[78] C.-Q. Geng, C.-C. Lee, and E. N. Saridakis, "Observational constraints on teleparallel dark energy," Journal of Cosmology and Astroparticle Physics, vol. 2012, no. 1201, p. 002, 2012.

[79] G. G. L. Nashed, "Monte Carlo evaluation of the external gamma, neutron and muon induced background sources in the CUORE experiment," Astroparticle Physics, vol. 2010, no. 330, p. 173, 2010.

[80] Y.-P. Wu and C.-Q. Geng, "Matter density perturbations in modified teleparallel theories," Journal of High Energy Physics, vol. 2012, no. 11, p. 142, 2012.

[81] L. Pogosian and A. Silvestri, "Erratum: Pattern of Growth in Viable," Physical Review D: Particles, Fields, Gravitation and Cosmology, vol. 77, no. 4, Article ID 023503, 2008.

[82] S. Nojiri and S. D. Odintsov, "Unified cosmic history in modified gravity: from $F(R)$ theory to Lorentz non-invariant models," Physics Reports, vol. 505, pp. 59-144, 2011.

[83] A. De Felice and S. J. Tsujikawa, " $\mathrm{f}(\mathrm{R})$ Theories," Living Reviews in Relativity, vol. 13, 2010.

[84] S. Capozziello and M. de Laurentis, "Extended Theories of Gravity," Physics Reports, vol. 509, no. 4-5, pp. 167-321, 2011.

[85] T. P. Sotiriou, "The nearly Newtonian regime in Non-Linear Theories of Gravity," Gen. Rel. Grav, vol. 38, 2006.

[86] V. Faraoni, "Erratum: Solar system experiments do not yet veto modified gravity models," Physical Review D: Particles, Fields, Gravitation and Cosmology, vol. 74, no. 2, Article ID 029902, 2006.
[87] S. Nojiri, S. D. Odintsov, and M. Sami, "Modified gravity and its reconstruction from the universe expansion history," Physical Review D: Particles, Fields, Gravitation and Cosmology, vol. 74, Article ID 086005, 2006.

[88] I. Sawicki and W. Hu, "Stability of cosmological solutions in $f(R)$ models of gravity," Physical Review D: Particles, Fields, Gravitation and Cosmology, vol. 75, Article ID 127502, 2007.

[89] Á. de la Cruz-Dombriz and A. Dobado, Physical Review D: Particles, Fields, Gravitation and Cosmology, vol. 74, no. 8, 2006.

[90] P. K. S. Dunsby, E. Elizalde, R. Goswami, S. Odintsov, and D. S. Gomez, " $\Lambda \mathrm{CDM}$ universe in $f(R)$ gravity," Physical Review $D$ : Particles, Fields, Gravitation and Cosmology, vol. 82, Article ID 023519, 2010.

[91] A. Sheykhi, "Higher-dimensional charged $f(R)$ black holes," Physical Review D: Particles, Fields, Gravitation and Cosmology, vol. 86, no. 2, Article ID 024013, 2012.

[92] A. Sheykhi, S. Salarpour, and Y. Bahrampour, "Rotating black strings in $\mathrm{f}(\mathrm{R})$-Maxwell theory," Physica Scripta, vol. 87, no. 4, Article ID 045004, 2013.

[93] S. Nojiri and S. D. Odintsov, "Anti-evaporation of Schwarzschild-de SITter black holes in $F(R)$ gravity," Classical and Quantum Gravity, vol. 30, no. 12, Article ID 125003, 2013.

[94] M. Hassaine and C. Martínez, "Higher-dimensional black holes with a conformally invariant Maxwell source," Physical Review D: Particles, Fields, Gravitation and Cosmology, vol. 75, no. 2, Article ID 027502, 2007.

[95] G. Cognola, E. Elizalde, S. Nojiri, S. D. Odintsov, and S. Zerbini, "One-loop $f(R)$ gravity in de Sitter universe," Journal of Cosmology and Astroparticle Physics, vol. 2005, no. 2, p. 10, 2005.

[96] T. Koivisto and H. Kurki-Suonio, "Cosmological perturbations in the Palatini formulation of modified gravity," Classical and Quantum Gravity, vol. 23, no. 7, pp. 2355-2369, 2006.

[97] L. Pogosian and A. Silvestri, "Erratum: Pattern of growth in viable," Physical Review D: Particles, Fields, Gravitation and Cosmology, vol. 77, no. 4, Article ID 023503, 2010.

[98] A. Nunez and S. Solganik, arXiv:hep-th/0403159.

[99] W. Hu and I. Sawicki, "Parametrized post-Friedmann framework for modified gravity," Physical Review D: Particles, Fields, Gravitation and Cosmology, vol. 76, Article ID 064004, 2007.

[100] Y. N. Obukhov and G. F. Rubilar, "Fresnel analysis of wave propagation in nonlinear electrodynamics," Physical Review D: Particles, Fields, Gravitation and Cosmology, vol. 74, 2006.

[101] W. Kopzyn'ski, "Variational principles for gravity and fluids," Annals of Physics, vol. 203, p. 308, 1990.

[102] A. Komar, "Asymptotic covariant conservation laws for gravitational radiation," Physical Review A: Atomic, Molecular and Optical Physics, vol. 127, no. 4, pp. 1411-1418, 1962.

[103] T. Shirafuji, G. G. Nashed, and Y. Kobayashi, "Equivalence principle in the new general relativity," Progress of Theoretical and Experimental Physics, vol. 96, no. 5, pp. 933-947, 1996.

[104] A. Komar, "Covariant conservation laws in general relativity," Physical Review A: Atomic, Molecular and Optical Physics, vol. 113, pp. 934-936, 1959.

[105] J. Winicour, "Angular momentum in general relativity," in General Relativity and Gravitation, A. Held, Ed., pp. 71-96, Plenum, New York, NY, USA, 1980.

[106] A. Ashtekar, "Angular momentum of isolated systems in general relativity," in Cosmology and Gravitation, P. G. Bergmann and V. de Sabbata, Eds., vol. 58, Plenum, New York, NY, USA, 1980. 
[107] I. Brevik, S. Nojiri, S. D. Odintsov, and L. Vanzo, "Entropy and universality of the Cardy-Verlinde formula in a dark energy universe," Physical Review D: Particles, Fields, Gravitation and Cosmology, vol. 70, no. 4 A, Article ID 43520, 2004.

[108] J.-X. Mo, G.-Q. Li, and X.-B. Xu, "Combined effects of $\mathrm{f}(\mathrm{R})$ gravity and conformally invariant Maxwell field on the extended phase space thermodynamics of higher-dimensional black holes," Eur. Phys. J. C, vol. 76, p. 545, 2016. 

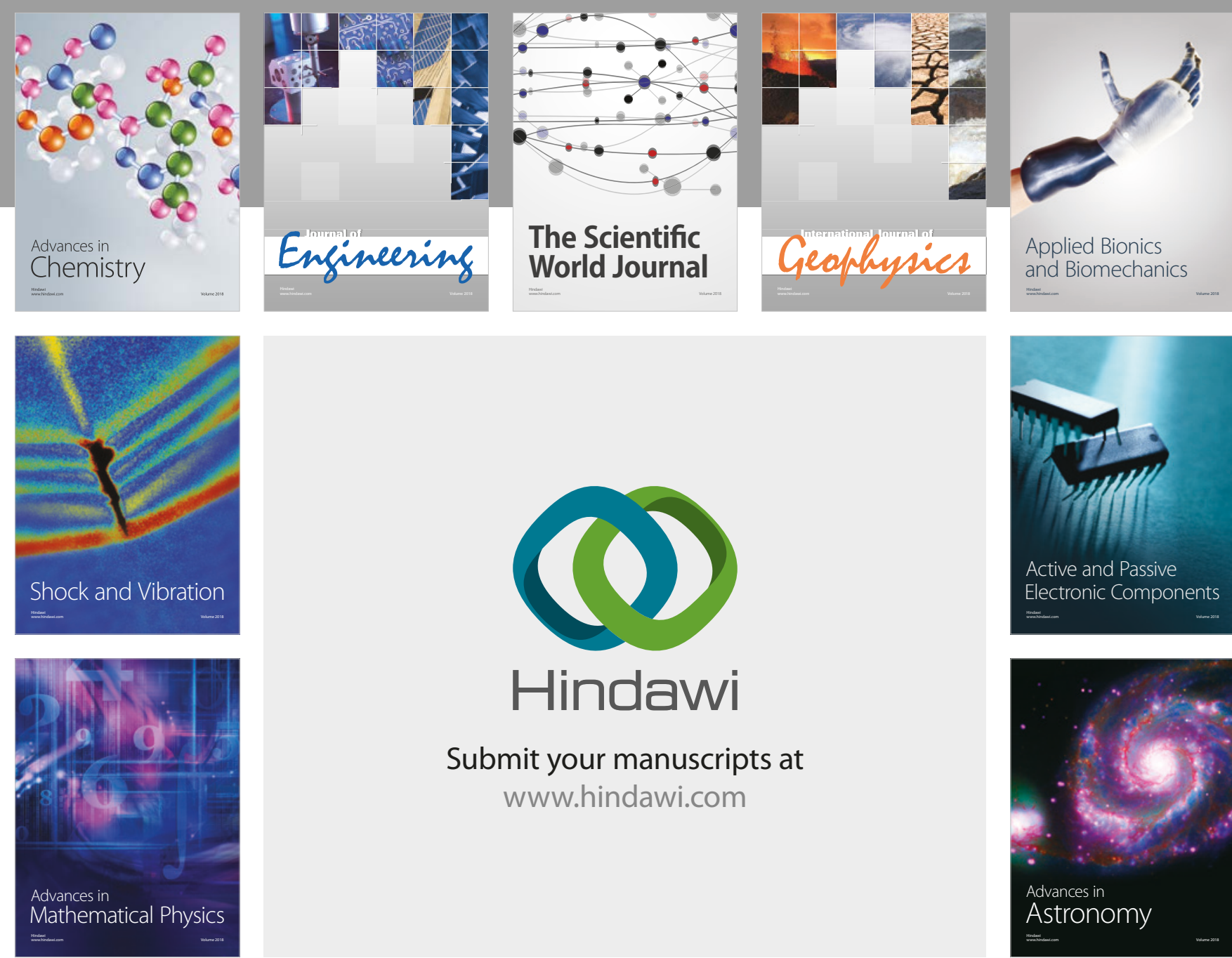

Submit your manuscripts at

www.hindawi.com

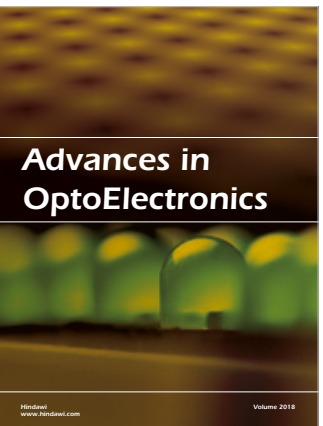

\section{Rotcting Machinery}
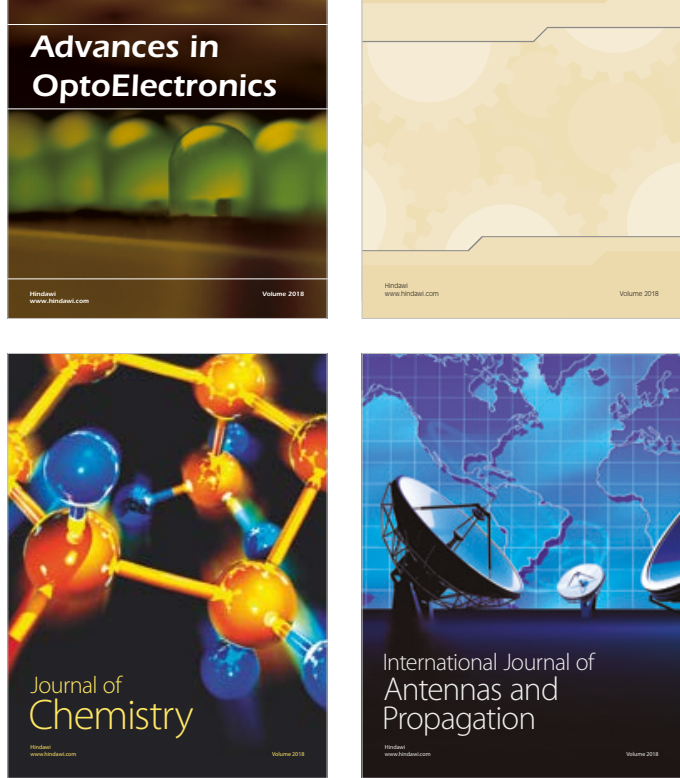

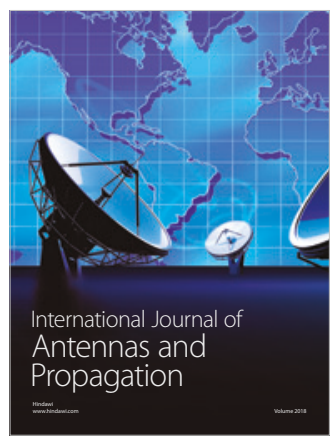

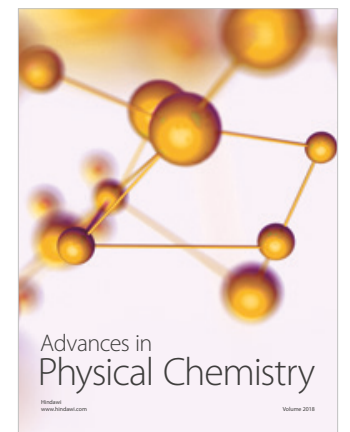

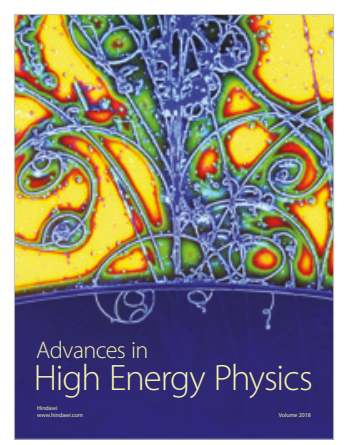

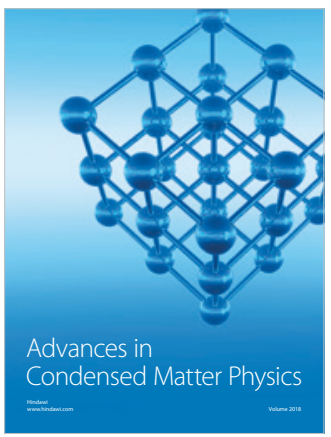

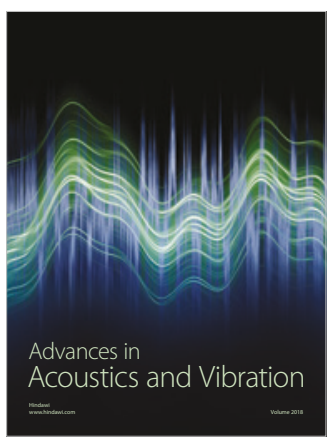

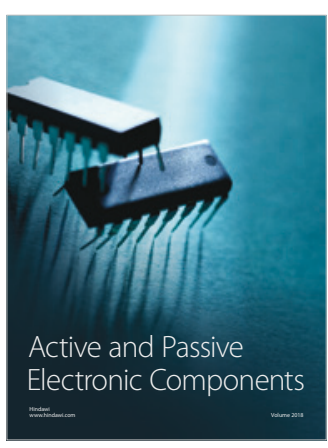
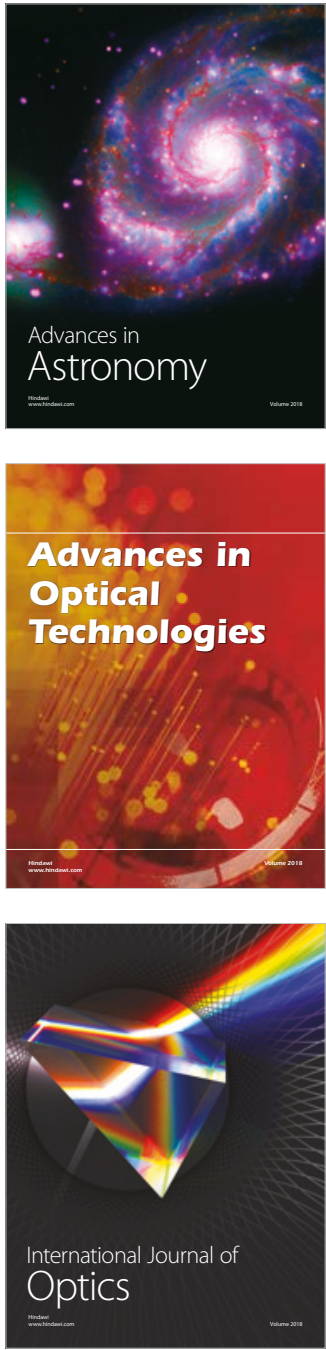